\title{
Acute Abdomen Misdiagnosed as Leaking Aortic Abdominal Aneurysm: An Unusual Presentation of Pulmonary Embolism: Case Report
}

\author{
Khater $\mathrm{E}^{1}$, Osman $\mathbf{M}^{2 *}$, Ibrahem $\mathbf{M}^{3}$ and Elkady $\mathbf{A}^{4}$ \\ ${ }^{1}$ Gastroenterology Department, Queen Elizabeth Hospital, \\ Birmingham, UK \\ ${ }^{2}$ General Surgery Department, Rotherham General \\ Hospital, Rotherham, UK \\ ${ }^{3}$ Radiology Department, Rotherham General Hospital, \\ Rotherham, UK \\ ${ }^{4}$ Birmingham Women's and Children's NHS Foundation \\ Trust, Birmingham, UK \\ *Corresponding author: Mohamed Osman, The \\ Rotherham NHS Foundation Trust, 19 Oakwood Hall \\ Drive, S60 3AQ, Rotherham, UK
}

Received: July 27, 2021; Accepted: September 23, 2021; Published: September 30, 2021

\begin{abstract}
We are presenting a case study about a 62-year-old lady who presented to A\&E with abdominal pain and hypotension. Initially her presentation was thought as leaking aortic aneurysm giving that all investigations done were inconclusive. CTAA was done accordingly which showed incidental finding of Pulmonary Embolism (PE). In Literature, we found few cases reported for $\mathrm{PE}$ with abdominal pain. Yet, our case discussed a unique presentation of PE with lower abdominal pain without desaturation of oxygen rather than upper abdominal pain with low oxygen saturation. Our case is supported with images of the CTPA and Chest X-ray. Our recommendation is that clinicians should consider PE as one of the differential diagnoses of acute abdomen due to its high mortality rate when misdiagnosed or missed diagnosed.
\end{abstract}

Keywords: Pulmonary embolism; Abdominal pain; CTPA

\section{Introduction}

Pulmonary Embolism (PE) is a highly fatal condition mostly because of the high incidence of missed diagnosis or misdiagnosis. Early diagnosis and management are the key factors to reducing mortality rates of PE. The most common symptoms of PE are dyspnoea, chest pain, cough, and haemoptysis [1]. Differential diagnosis of acute abdomen and abdominal pain in general is diverse, yet pulmonary embolism is not one of them. However, this needs to be changed. We report an unusual presentation of $\mathrm{PE}$ as our patient presented with abdominal pain referred to the back and hypotension. Our case was initially misdiagnosed as leaking aortic abdominal aneurysm. In this case report, the patient presented to accident and emergency department for abdominal pain without the typical symptoms of PE such as chest tightness, shortness of breath and dyspnoea. She was initially misdiagnosed as leaking aortic abdominal aneurysm until the CTAA report showed PE as an incidental finding.

\section{Case Presentation}

A 62-year-old female presented to emergency department with lower abdominal pain referred to the back only relieved by defecation for 4 days. There was guarding and rigidity in abdominal examination, especially in the suprapubic and umbilical area. Vital signs initially were within normal limits apart from low blood pressure of 105 systolic and 76 diastolic. Cardiopulmonary auscultation and abdominal bed side ultrasound scan were both unremarkable. Her medical and social history were only significant for liver failure and heavy chronic smoking. Blood amylase, lipase, liver function, and blood routine including arterial gas were all not concerning. Investigations done including chest x-ray and ecg did not show any abnormality. Given that the abdominal pain is referred to the back with hypotension, the preliminary diagnoses of leaking abdominal aortic aneurysm was considered. Computerized Tomography Aortogram Angiography (CTAA) was done to rule aortic abnormalities and differentials of acute abdomen. CT showed no evidence of aortic dissection or aneurysm, major abdominal organs grossly appeared unremarkable, no enlarged abdominal nodes, uncomplicated colon diverticulae, no dilated bowel loops and no free fluid in abdomen or pelvis. Unexpectedly, findings were consistent with pulmonary embolism. The patient was admitted to the hospital and received low molecular weight heparin for anticoagulant therapy. Her abdominal pain was significantly relieved afterwards. To the best of our knowledge, our case presents a unique and atypical presentation not only because of presenting with only abdominal pain and no typical symptoms of PE but also because the pain was in lower abdomen and oxygen saturation was normal. In other cases, low oxygen saturation and site of abdominal pain which is mostly upper abdominal was the key to the PE diagnosis (Figure 1 and 2).

\section{Discussion}

In literature, abdominal pain is reported in few patients with PE. In fact, some studies have begun to suggest that PE should be considered for upper abdominal pain [2]. In Japan, the 2015 guidelines for acute abdomen states that PE should be considered

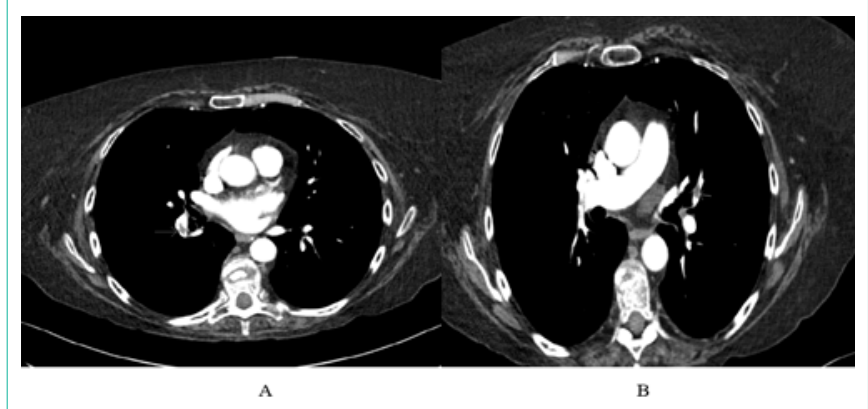

Figure 1: Filling defects in right and left upper interlobar pulmonary arteries compatible with peripheral pulmonary emboli (white arrows).
Austin J Radiol - Volume 8 Issue 9 - 2021

ISSN : 2473-0637 | www.austinpublishing group.com

Khater et al. () All rights are reserved
Citation: Khater E, Osman M, Ibrahem M and Elkady A. Acute Abdomen Misdiagnosed as Leaking Aortic Abdominal Aneurysm: An Unusual Presentation of Pulmonary Embolism: Case Report. Austin J Radiol. 2021; 8(9): 1161. 


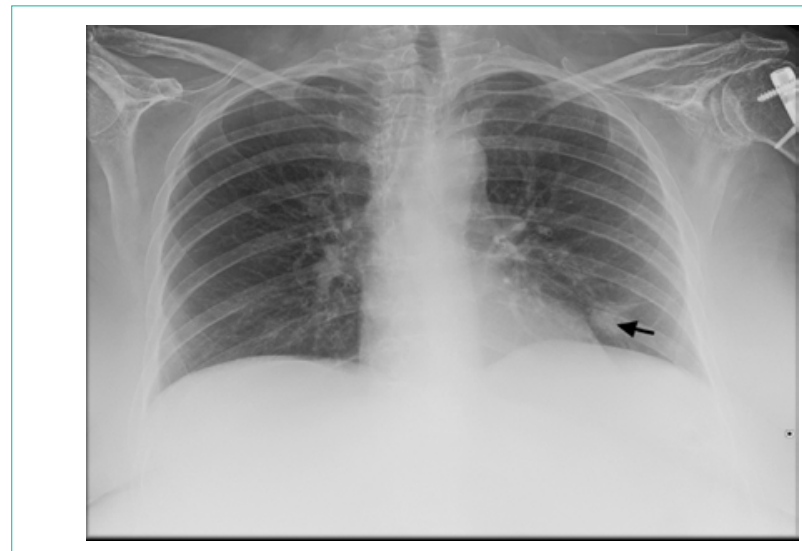

Figure 2: Consolidation in left lower lung zone in keeping with infection (black arrow).

as part of the differentials [3]. As PE can cause right sided heart failure, liver capsule or gall bladder dilatation may occur thus causes abdominal pain [4]. There was a large study in 2011, in which around 11 percent of 1880 patients diagnosed with PE had upper abdominal pain [5]. Another possible cause for the abdominal pain could be that the increased viscosity of blood with low oxygen may lead to a small embolus that may cause focal necrosis of abdominal organs [6]. Also, pulmonary hypertension may lead to abdominal lymphedema and hepatobiliary portal infiltration [7]. Alternatively, the abdominal pain may be caused by the tension of sensory nerve endings within the lower part of the chest wall parietal pleura and intercostal muscle hyperalgesia [8].

\section{Conclusion}

In patients with abdominal pain, clinicians do not think of the possibility of PE. From our case report and other studies in literature, $\mathrm{PE}$ should be considered in the differential diagnosis especially in those with risk factors or high suspicions. We recommend arterial blood gas and $\mathrm{D}$-dimer to be performed to patients presenting with acute abdomen and then correlate clinically with the results.

\section{References}

1. Konstantinides SV, Torbicki A, Agnelli G, et al. 2014 ESC guidelines on the diagnosis and management of acute pulmonary embolism. Eur Heart $\mathrm{J} 2014$; 35: 3033-3069; 3069a-3069k.

2. Tapson VF. Acute pulmonary embolism. N Engl J Med. 2008; 358: 10371052.

3. Stein PD, Beemath A, Matta F, et al. Clinical characteristics of patients with acute pulmonary embolism: data from PIOPED II. Am J Med. 2007; 120: 871879.

4. Cartwright SL, Knudson MP. Evaluation of acute abdominal pain in adults. Am Fam Physician. 2008; 77: 971-978.

5. Fields JM, Dean AJ. Systemic causes of abdominal pain. Emerg Med Clin North Am. 2011; 29: 195-210.

6. Mayumi T, Yoshida M, Tazuma S, et al. The practice guidelines for primary care of acute abdomen 2015. Jpn J Radiol. 2016; 34: 80-115.

7. Mayumi T, Yoshida M, Tazuma S, et al. The practice guidelines for primary care of acute abdomen 2015. Jpn J Radiol. 2016; 34: 80-115.

8. Pollack CV, Schreiber D, Goldhaber SZ, et al. Clinical characteristics, management, and outcomes of patients diagnosed with acute pulmonary embolism in the emergency department: initial report of EMPEROR (Multicenter Emergency Medicine Pulmonary Embolism in the Real World Registry). J Am Coll Cardiol. 2011; 57: 700-706.

9. von Pohle WR. Pulmonary embolism presenting as acute abdominal pain. Respiration. 1996; 63: 318-320.

10. Morel J, Mahul P, Cuilleron M, et al. Mediastinal and abdominal lymphoedema: consequence of massive pulmonary embolism with shock? Ann Fr Anesth Reanim. 2004; 23: 142-145.

11. Gorham LW. A study of pulmonary embolism. III. The mechanism of pain; based on a clinicopathological investigation of 100 cases of minor and 100 cases of massive embolism of the pulmonary artery. Arch Intern Med. 1961; 108: 418-426. 